decreases as $\lambda$ increases. Moreover when $\lambda$ is sufficiently large, as is well known, $y$ and consequently $V$ have as many roots as desired when $a<x<b$. Using these facts and Theorems I and II we now readily prove the following:

Theorem III. There exist unique values, $\lambda_{1}<\lambda_{2}<\lambda_{3}$ $<\cdots$, such that when $\lambda=\lambda_{j}, j=1,2,3, \cdots$, a solution $y$ of (3) exists satisfying (4) such that $V(x)$ has exactly $j$ roots on the interval $a<x<b$.

We can extend this to $j=0$ if, when $G=0, V$ has no root on the interval $a<x<b$.

The University of Alabama, September, 1917.

\title{
NOTE ON INFINITE SYSTEMS OF LINEAR EQUATIONS.
}

BY DR. W. L. HART.

(Read before the American Mathematical Society April 28, 1917.)

IN considering infinite systems of linear equations

$$
\sum_{j=1}^{\infty} a_{i j} x_{j}=x_{i}{ }^{\prime} \quad(i=1,2, \cdots),
$$

particular interest is attached to those whose solutions preserve the properties of the solutions of a set of $n$ linear equations in $n$ variables. It is known* that the system (1) possesses this property if $a_{i j}=d_{i j}-b_{i j}\left(d_{i i}=1 ; d_{i j}=0, i \neq j\right)$, where the infinite matrix $B=\left(b_{i j}\right)_{i, j=1,2}, \ldots$ is completely continuous. $\dagger$ The discussion of Riess deals only with the special case $a_{i j}=d_{i j}-b_{i j}$, but it is easily found that his proof holds for the more general case stated below. The proof of the theorem of this note is not given since it differs only in minor details from the proof of the theorem given by Riess.

It will be said that a matrix $A_{1}$ is a sub-matrix of the matrix

* Cf. F. Riess, Equations Linéaires, p. 94.

$\dagger$ Cf. F. Riess, loc. cit. 
$A=\left(a_{i j}\right)_{i, j=1,2}, \ldots$ in case it is obtained from $A$ by striking out certain $k$ rows and $k$ columns ( $k$ finite).

Let the system (1) be taken in the field of complex numbers and suppose that the sets of numbers $x=\left(x_{1}, x_{2}, \cdots\right)$ and $x^{\prime}=\left(x_{1}^{\prime}, x_{2}^{\prime}, \cdots\right)$ represent points in Hilbert space; that is, $\sum_{i=1}^{\infty}\left|x_{i}\right|^{2}$ and $\sum_{i=1}^{\infty}\left|x_{i}\right|^{2}$ converge. The main result of the present note is

Theorem I. Let the matrix $A=\left(a_{i j}\right)_{i, j=1,2}, \ldots$ be limited in the sense of Hilbert.* Suppose there exists a sub-matrix $A_{1}$ possessing a unique limited reciprocal $A_{1}^{-1}$. Then, the system of linear equations (1) preserves the characteristics of a system of $n$ linear equations in $n$ unknowns.

That is, either (a) for every $x^{\prime}$ system (1) possesses a unique solution, or (b) there is an integer $h>0$ such that if $x^{\prime}$ is a point in Hilbert space satisfying certain $h$ linear relations, there exists a solution of (1) depending linearly on $h$ parameters.

With the help of certain details necessary for the proof of Theorem I, there is easily proved

Theorem II. If the matrix $A$ satisfies the hypotheses of Theorem I and if the homogeneous system

$$
\sum_{j=1}^{\infty} a_{i j} x_{j}=0 \quad(i=1,2, \cdots)
$$

has no solution in Hilbert space except $x=0$, then $A$ possesses a unique limited reciprocal matrix.

The proof is simple because the reciprocal matrix of the theorem is actually constructed during the proof of Theorem I.

For the system (1) with $a_{i j}=d_{i j}-b_{i j}$ where $B=\left(b_{i j}\right)$ is completely continuous, the hypothesis of Theorem $\mathrm{I}$ is always satisfied by taking, as the sub-matrix $A_{1}$, the infinite matrix obtained by striking out the first $n$ rows and $n$ columns where $n$ is taken sufficiently large. Such a matrix is known $\dagger$ to have a unique limited reciprocal matrix.

83RD ARTILLERY,

Fort D. A. Russeli, Wyoming, November 10, 1917.

* Hellinger and Toeplitz, Math. Annalen, vol. 69 (1910), p. 299.

† Riess, loc. cit., p. 98. 